\title{
A Review of some recent advancements in Non-Ideal Compressible Fluid Dynamics
}

\author{
Giulio Gori ${ }^{1,2}[0000-0001-7442-2773]$, Olivier Le Maitre ${ }^{1,2,3[0000-0002-3811-7787]}$, \\ and Pietro M. Congedo ${ }^{1,2}[0000-0003-3266-3549]$ \\ 1 Centre de Mathématiques Appliquées, École Polytechnique, IPP, Route de Saclay, \\ 91128 Palaiseau, France \\ 2 INRIA \\ 3 CNRS \\ giulio.gori@inria.fr
}

\begin{abstract}
This paper reviews selected advancements concerning the theoretical, numerical and experimental investigation of Non-Ideal Compressible Fluid Dynamics (NICFD) flows for Renewable Energy applications. First, we review the so-called non-ideal oblique shock waves. After, we briefly discuss the state-of-the-art concerning computational models for NICFD applications, with a particular focus on the predictions accuracy assessment. Eventually, we describe a Bayesian framework for inferring the material-dependent parameters appearing in complex thermodynamics models for NICFD.
\end{abstract}

Keywords: NICFD · Uncertainty Quantification · UTOPIAE · NSHOCK.

\section{Introduction}

Non-Ideal Compressible-Fluid Dynamics (NICFD) is devoted to the investigation of compressible flows endowed with a particular thermodynamic behavior. Namely, thermodynamics does not abide by the equation of state for ideal gases, $P=\rho R T$ with $P$ being the pressure, $\rho$ the fluid density, $R$ the gas constant and $T$ the fluid temperature. These are molecular complex vapor flows operating in thermodynamics conditions close to the liquid-vapor saturation curve and critical point. In the non-ideal state, flows may exhibit peculiar features such as a non-ideal increase of the speed of sound upon isentropic expansion, see $[2,48$, $41,23,43]$. The qualitative behavior of compressible-fluid flows is classified by means of the so-called Fundamental Derivative of Gasdynamics $\Gamma$ [41]

$$
\Gamma \equiv \frac{c^{4}}{2 v^{3}}\left(\frac{\partial^{2} v}{\partial P^{2}}\right)_{s}, \quad \text { with } \quad c^{2} \equiv\left(\frac{\partial \mathrm{P}}{\partial \rho}\right)_{\mathrm{s}},
$$

where $s$ the entropy, $v$ the specific volume and $c$ is the speed of sound.

Conventional treatments of gasdynamics assume, either explicitly or implicitly, that $\Gamma>1$ in the dilute gas limit. From the qualitative point of view, the gasdynamic behavior of any fluid with constant $\Gamma>1$ resembles that of 
ideal gases. In the close proximity of the vapor-liquid saturation curve, the fluid can show values of $\Gamma$ smaller than one, therefore being non-ideal. It is standard practice to distinguish between the cases $\Gamma \leq 0$ and $0<\Gamma \leq 1$ : the former case refers to non-classical gasdynamics, as opposed to classical gasdynamics for which $\Gamma>0$. If present, the extension of the non-ideal and non-classical region vary from fluid to fluid and depending on the thermodynamic model employed. Within the non-classical limit, unconventional phenomena such as expansion shocks, isentropic compression fans, split shocks and composite waves are allowed, see for instance references [41, 43, 8-10, 25, 1]. The so-called non-classical phenomena are caused by negative non-linearities ( $\Gamma$ changing its sign), and the most striking example is the admissibility of expansion shocks and composite waves. It must be noticed, though, that experimental evidence of negative nonlinearities is still missing at present, thus leaving the existence of non-classical phenomena an open question in fluid mechanics. In this respect, recent advancements indicate the possibility of observing negative nonlinearities in common substances (other than Bethe-Zel'dovich-Thompson fluids) due to critical-point anomalies $[27,28]$. Nevertheless, these phenomena go beyond the scope of this contribution which is limited to classical flows only.

The compressibility factor $Z$ is a parameter that quantifies how much the flow departs from the ideal regime. $Z$ is defined as follows:

$$
Z=\frac{P v}{R T}
$$

In the ideal regime the compressibility factor is equal (or close) to unity; as the thermodynamics departs from ideality, $Z$ decreases.

In this contribution, we presented a review of the advancements achieved partially within the UTOPIAE project frame, a Marie Curie Innovative Training Network funded by the European Commission through the H2020 funding stream, and partially within the frame of the European Research Council (ERC) Consolidator Grant project NSHOCK. Moving from the discussion of an unprecedented physical phenomenon presented in Sec. 2, we review the very first accuracy assessment of computational models for NICFD flows in Sec. 3 and an observation-based inferential investigation of the material-dependent parameters included in complex thermodynamics models, in Sec. 4.

\section{Non-Ideal Oblique Shock Waves}

In this section, we discuss non-ideal gasdynamic effects in steady oblique shockwaves. Part of the contents presented hereinafter appear in Ref. [17, 18, 46, 44, $50,49,15,45,11]$.

In particular, we review the so-called non-ideal oblique shock waves i.e., classical compression shocks of finite amplitude across which a Mach number increase is observed $[17,18,46,15,45]$. Across a shock, the fluid undergoes a transformation from state $A$ to state $B$. In polytropic dilute gases, the post-shock thermodynamic and kinematic quantities, and the wave angle itself, are completely 
determined by the Mach number of the flow ahead of the shock and by the specific heat ratio $\gamma$.

In [17], it was shown that a non-ideal vapor suffers from a more or less noticeable dependence on the pre-shock thermodynamic state. Furthermore, there exists a particular set of pre-shock states resulting into a non-ideal Mach number increase across the wave. This peculiar kind of waves are referred to as non-ideal oblique shocks. The definition of their thermodynamic admissibility region and the extension of the mathematical model to flows of fluids exhibiting positive non-linearity can be found in [46]. From the theoretical point of view non-ideal oblique shocks are possible for any flow in the regime $\Gamma<1$. Numerical experiments, presented in $[17,46]$, were performed using the polytropic van der Waals model of siloxane fluid MDM. In some cases, the post-shock Mach number was found to increase up to 1.5 times the pre-shock Mach number. In Fig. 1(a-b), we report the variation of the pre-post shock ratio of some quantities of interest, for two of the conditions reported in [17]. The selected configurations exhibit the same upstream values of the entropy and of the Mach number $\left(s_{A}, M_{A}\right)$. By retaining the labeling defined in [17], Fig. 1(a) reports results related to state $A_{1}$, with $\Gamma_{A_{1}}>1$. Since $\Gamma_{A_{1}}>1$, the model predicts $M_{B}<M_{A}$ for each possible oblique shock configuration with upstream state $A_{1}$. Note that suffix $n$ identifies the quantity component normal to the shock front.

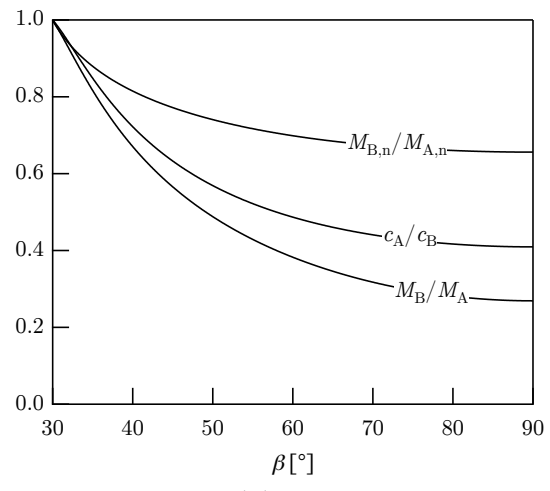

(a)

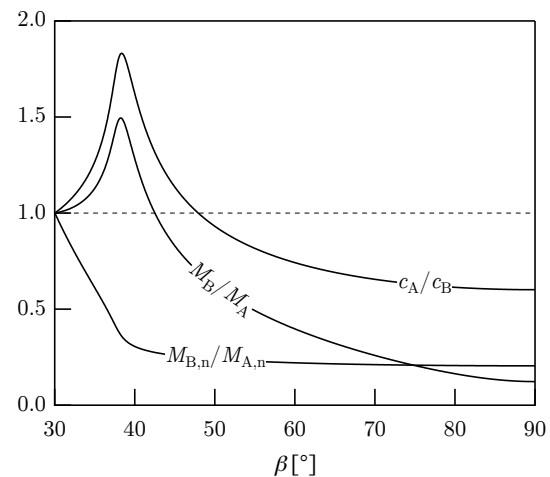

(b)

Fig. 1. Variation of the speed of sound ratio $c_{A} / c_{B}$, normal Mach number ratio $M_{B, n} / M_{A, n}$ and Mach number ratio $M_{B} / M_{A}$ with shock angle $\beta$ as computed from the polytropic vdW model siloxane fluid MDM. The shock angle spans from the minimum value $\beta=\operatorname{asin}\left(1 / M_{A}\right)$, corresponding to an acoustic wave, up to the maximum value $\beta=\pi / 2$, corresponding to a normal shock wave. (a) case $A_{1}$; (b) case $A_{2}$;

In Fig. 1(b), state $A_{2}$, with $\Gamma_{A_{2}}<1$ is considered showing that there exists a range of shock angles in which the Mach number increases. Due to the almost negligible entropy rise, and together with $\Gamma_{B}<1, c_{B}$ initially decreases with increasing shock angle, as shown in Fig. 1(b), where $c_{B}<c_{A}$ up to 47.9 [deg]. 
A pronounced local minimum if found at 38.3 [deg]. The post-shock state characterized by $M_{B}=M_{A}$ occurs at 42.5 [deg] whereas the local peak for $M_{B}$ is found at $38.2[\mathrm{deg}]$ and corresponds to the ratio $M_{B} / M_{A}=1.5$.

The variation of the flow deflection angle $\theta$ with the shock angle $\beta$ for each of the configurations considered is shown in Fig. 2(a), where it is compared with the curve labelled $I G$, which corresponds to the polytropic ideal gas case and, as is well-known, it is independent of the pre-shock thermodynamic state $P_{A}, v_{A}$ [42]. The curves computed by means of the polytropic vdW model exhibit substantial

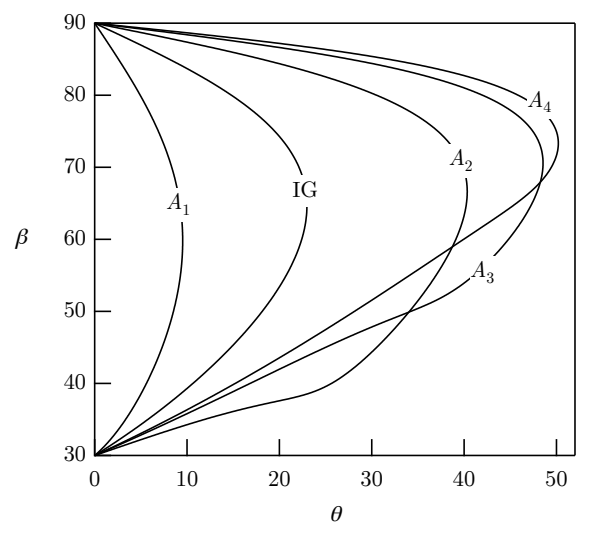

(a)

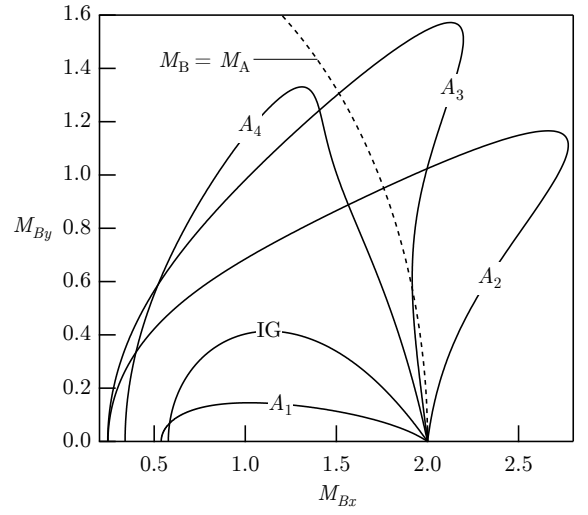

(b)

Fig. 2. Diagrams illustrating (a) the variation of the flow deflection angle $\theta$ with shock angle $\beta$ and (b) the polar of the Mach number, where $M_{B x}=M_{B} \cos \theta$ and $M_{B y}=$ $M_{B} \sin \theta$, as computed from the polytropic van der Waals model of siloxane fluid MDM. Also shown are the curves, labeled $I G$, that correspond to the polytropic ideal gas case with the same upstream Mach number $M_{A}$ used in the computation of the non-ideal cases.

differences depending on the pre-shock thermodynamic state. In particular, the maximum turning angle $\theta_{\max }$ that the flow can sustain across a planar attached shock wave varies from 9.5 [deg] of case $A_{1}$ up to 50.2 [deg] of case $A_{4}$. In contrast, $\theta_{\max }=23.0[\mathrm{deg}]$ for the polytropic ideal gas.

Finally, the same results can be conveniently presented also in term of the shock polar for the Mach number, namely a plot of $M_{B y}=M_{B} \sin \theta$ versus $M_{B x}=M_{B} \cos \theta$, as shown in Fig. 2(b) for each non-ideal configuration considered and the polytropic ideal gas counterpart. This analysis has a sound theoretical basis, owing to the fact that the simple van der Waals model is known to predict the correct qualitative behavior in the single-phase thermodynamic region close to liquid-vapour equilibrium (sufficiently far from the critical point for critical phenomena to be negligible). Moreover, the occurrence of non-ideal shock waves is confirmed for diverse substances using also state-of-the-art thermodynamic models, see [18, 46, 45]. 
The experimental verification of the occurrence of non-ideal shock waves can be devised in test rigs working with fluids of high or even moderate molecular complexity. In $[50,49]$, steady oblique shock waves were observed for the first time in non-ideal supersonic flows of single-phase organic vapors. Oblique shock waves were observed and characterized experimentally at varying stagnation conditions in the pre-shock state, for a set of different flow deviation angles. Observations confirm the shock wave theory for two-dimensional steady flows, proving a purely non-ideal dependence, in addition to the well-known dependence on the preshock Mach number, specific heat ratio and flow deviation angle typical of dilute gas conditions, of the shock pressure ratio on stagnation conditions. Figure 3(a) reports the Schlieren observation of oblique shocks propagating in a supersonic non-ideal stream. The stream is obtained using a planar converging-diverging nozzle. A backward facing step at the nozzle throat produces symmetric shocks propagating downtream, into the divergent section. The location of pressure probes is also indicated with green dots. Figure 3(b) reports instead the Schlieren observation of the shock pattern generated around a diamond-shaped airfoil profile plunged into a supersonic stream.

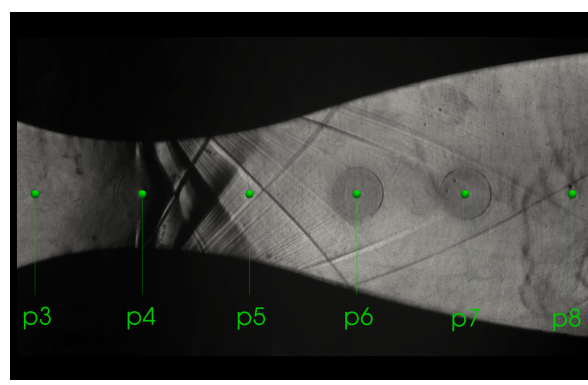

(a)

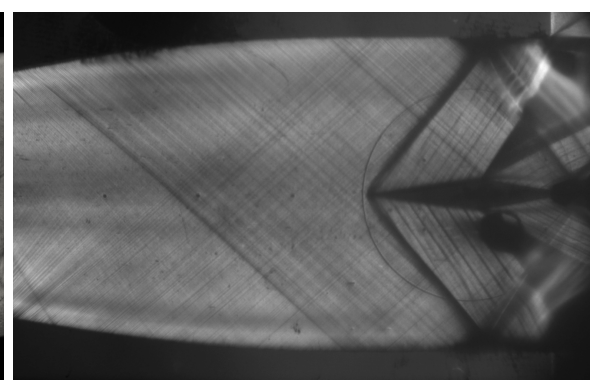

(b)

Fig. 3. Experimental Schlieren images of experiments for the observation of oblique shocks in non-ideal supersonic flows.

The non-ideal increase of the flow Mach number across oblique shocks and the dependence of the shock angle and flow deviation from the upstream thermodynamic state are arguably relevant in applications where oblique shock waves are either intentionally formed (e.g. engine intake ramps) or a byproduct of the supersonic flow expansion (e.g. fish-tail shocks in turbine nozzle vanes, over/underexpanded jet from nozzle exit). In Ref. [44] the authors investigate the relevance of non-ideal oblique shocks to renewable energy application.

\section{NICFD Computational Model Accuracy Assessment}

The SU2 an open-source suite $[29,12]$ embodies the reference among NICFD computational solvers. In the limited framework of ideal flows of air, the reli- 
ability of the SU2 suite was extensively assessed $[29,30,13,12,35]$. Preliminary verification of the SU2 NICFD solver implementation can be found in $[47,16$, $33]$. The results shown hereinafter are taken from $[21,19,49,15]$. These works, developed in the frame of the NSHOCK and UTOPIAE projects, concern the first-ever experimental assessment of a Non-Ideal Compressible-Fluid Dynamics (NICFD) model.

The experimental test set considered to carry out the assessment includes flow configurations of practical interest for renewable energy applications. More precisely, experiments aim at reproducing the supersonic flow of MDM within turbine vanes in ORC power production systems. These typical flow configurations, involving fluid flows in mildly-to-highly non-ideal regimes, are reproduced numerically, using the non-ideal solver from SU2. Results are then compared against pressure and Mach number measurements collected at the Compressiblefluid dynamics for Renewable Energy Applications (CREA) laboratory of Politecnico di Milano, using the Test-Rig for Organic VApours (TROVA) [37, 22, 32]. The TROVA is equipped with pressure probes, to record the value of static pressure at the test section side wall. Temperature and pressure are also monitored within the settling chamber, ahead of the test section. Mach number measurements are also available through the post-processing of schlieren images. Gaussian Probability Density Function (PDF) distribution are systematically considered for all the observations.

The computational model accuracy assessment takes advantage of an Uncertainty Quantification (UQ) analysis [34], to assess the role of aleatory uncertainties on the nominal test conditions and thus to quantify the validity and robustness of SU2 predictions. Uncertainties are propagated through the solver and statistical moments of selected outputs are computed using a non-intrusive Polynomial Chaos expansion approach [24]. The output statistics, complemented with their numerical $2 \sigma$ range, are compared against the experimental measurements and their associated tolerances. Several papers addressed the problem of quantifying uncertainties in the numerical simulation of non-ideal flows. Some of these papers $[3,4,26]$ specifically focused the attention on thermodynamic models. In other works $[6,7,14]$, multiple sources of uncertainties have been taken into account, both on operating conditions and thermodynamic models. According to the conclusions presented in these works, the uncertainties related to the thermodynamic model can be neglected with respect to the ones related to the operating conditions of the experimental facility. Therefore, in the following two sources of uncertainty were taken into account. Namely, the uncertainty on the measured values of the total pressure and total temperature at the inlet of the test section.

The test case and the results reported here are taken from [19]. The assessment test case considered in this review consists of a planar converging-diverging nozzle and the experiment aims at reproducing the isentropic expansion of a siloxane MDM flow in a non-ideal regime. Further details regarding the geometry and the test-rig set-up can be found in [39] and [36]. The TROVA is a blowdown facility and, as the high-pressure reservoir empties, the flow encompasses highly 
non-ideal to ideal regimes. In the original work [19], 5 time instances (A-E) were selected during two discharges at diverse reservoir conditions, for a total of 10 different flow configurations. Here, retaining the same labeling, we show only the $A_{1}, E_{1}$ and the $A_{2}, E_{2}$ cases.

These experiments were reproduced numerically by means of steady simulations of inviscid flows, using diverse meshes and diverse thermodynamic models. To assess the role of the viscous effects, two and three-dimensional simulations based on the Reynolds-averaged Navier-Stokes (RANS) equations were also carried out. Figure 4 shows the pressure mean trends, for the considered experiments ( $A_{1}-E_{1}$ on the left column and $A_{2}-E_{2}$ on the right column), complemented by $\pm 2 \sigma$ ranges resulting from the $\mathrm{UQ}$ analysis. On the same plots, experimental measurements (diamond marks) and their $2 \sigma$ uncertainty bars are reported for comparison.

The numerical solution fairly matches experimental data in all cases: the mean pressure trend is indeed very close to the measured values. Discrepancies are generally found near the exhaust section, where the increase of the boundary layer thickness causes the flow to re-compress. Moreover, differences reduce as the flow regime drifts from highly non-ideal to ideal, see [19].

Schlieren images are exploited to directly measure the local value of the Mach number for test $A_{2}$. Fig. 5(a) reports the experimental measurements and the related error bars, see [38]. In the same plot, the numerical mean solution and the $\pm 2 \sigma$ interval, resulting from the UQ analysis of the Mach number, for test $A_{2}$, are also reported. The mean solution is well included within the experimental error bars, pointing out the reliability of the predicted Mach number trend.

For test $A_{2}$, a sensitivity analysis based on the computation of the Sobol indices for static pressure is reported in Fig. 5.(b), Clearly, the analysis reveals that the inflow total pressure uncertainty utterly dominates. On the other hand, the inflow total temperature uncertainty contributes significantly to the static pressure variability in the close proximity of the discharging section.

\section{Bayesian Inference of Fluid Model Parameters}

The recent offspring of experimental facilities for the investigation of non-ideal flows goes so far as to envision that a considerable amount of data will be soon available. Certainly, this calls for the development of novel and reliable tools, to take advantage of data collected in experiments.

In this section, we report the description of a Bayesian framework for inferring the material-dependent parameters appearing in complex thermodynamics models for non-ideal flows. The contents presented hereinafter appear in Ref. [15, 20]. The framework is tailored on the TROVA test-rig at Politecnico di Milano $[37,22,32]$ and it is intended to evaluate the potential analysis which could be done on NICFD flows data. In particular, results reported hereinafter concern the inference of the Peng-Robinson (PR) fluid model parameters[31]. The calibration data set $\mathbf{o}$ is relative to the non-ideal expansion of a supersonic MDM flow across a converging diverging nozzle. The reader is referred to [15,20] for 

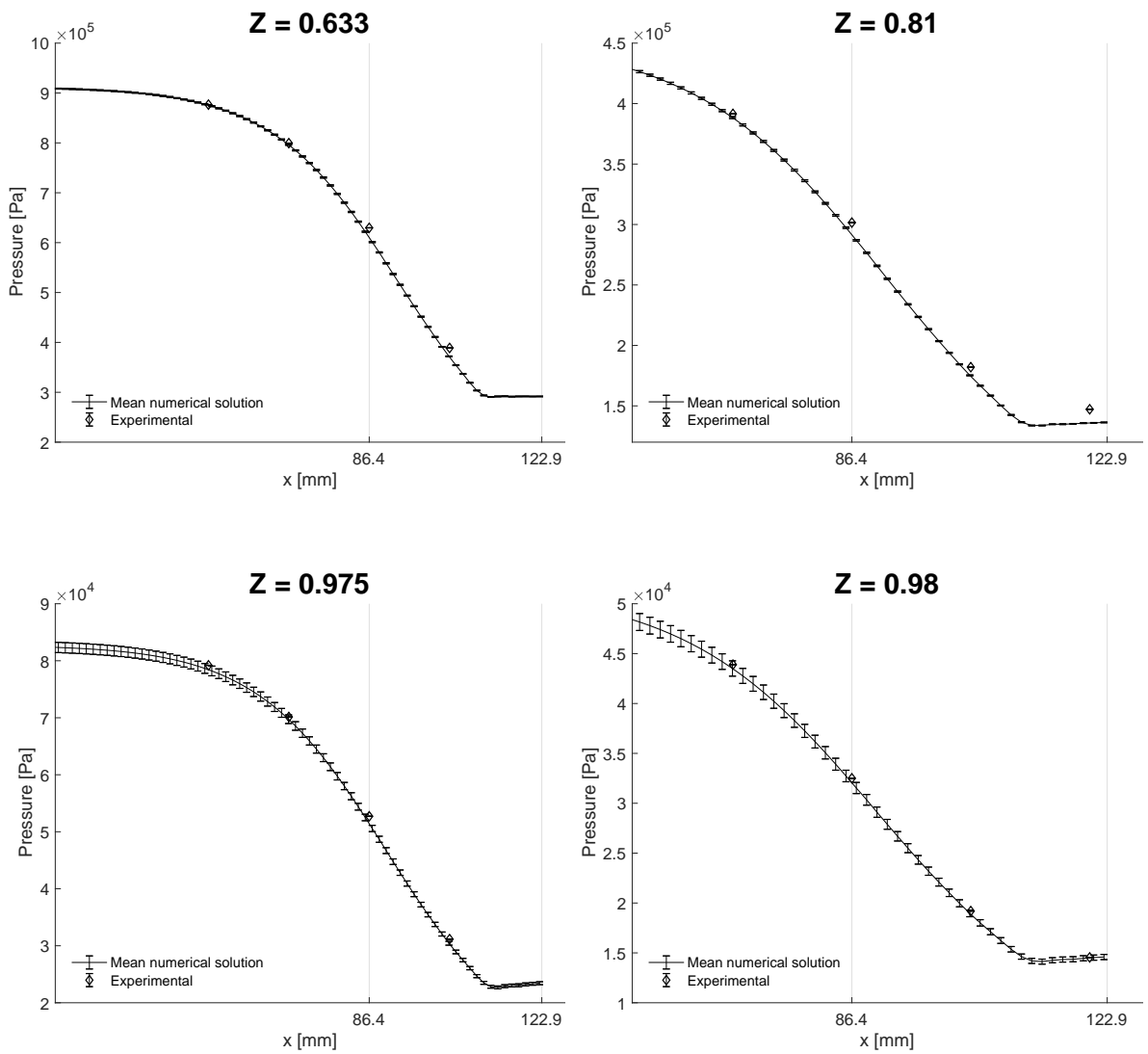

Fig. 4. accuracy assessment for discharge 1 (left column) and 2 (right column). Experiments $A$ (top row) and $E$ (bottom row). The continuous lines are the CFD predictions, complemented by the uncertainty bars. Diamond marks correspond to experimental measurements. 


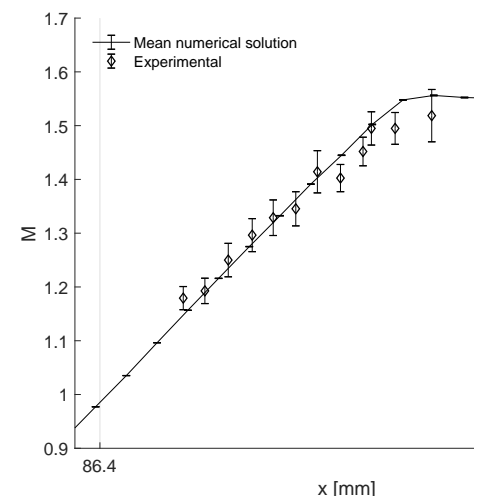

(a)

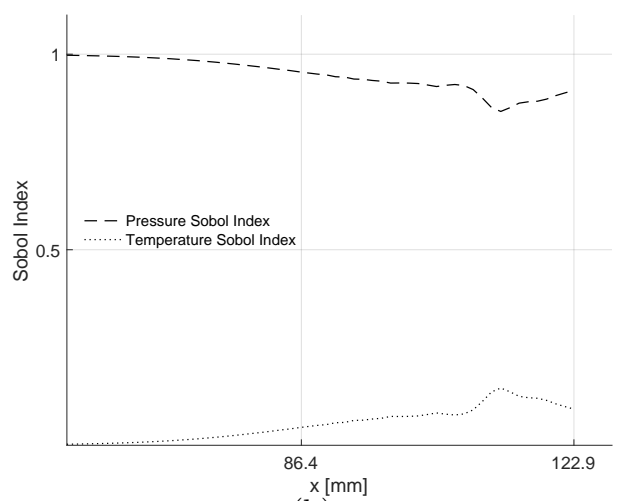

(b)

Fig. 5. case $A_{2}$. (a) Experimental values and error bars relative to the Mach number measure are compared against the mean solution and the numerical error bars resulting from the UQ analysis; (b) Sobol indices for static pressure related to uncertainty on both the values of total pressure and total temperature at the inlet.

a thorough description of the experiments and for a detailed summary of test conditions. In the inference process, the unknown variables set $\mathbf{q}$ includes the TROVA operating conditions and the PR model parameters. The operating conditions are included in the inferential process and treated as nuisance parameters. According to this notation, the Bayes theorem reads

$$
\mathcal{P}(\mathbf{q} \mid \mathbf{o}) \propto \mathcal{P}(\mathbf{o} \mid \mathbf{q}) \mathcal{P}(\mathbf{q}),
$$

being $\mathbf{q}=\left(P^{\mathrm{t}}, T^{\mathrm{t}}, P_{c r}, T_{c r}, \omega, \gamma\right)^{T}$, respectively, the inflow total pressure, the inflow total temperature, the MDM critical pressure and temperature, the MDM acentric factor and the specific heat ratio. A gaussian likelihood function $\mathcal{L}$ is employed. Uniformly distributed priors $\mathcal{P}(\mathbf{q}) \sim \mathcal{U}_{\mathbf{q}}\left[\mathbf{q}_{\text {min }}, \mathbf{q}_{\text {max }}\right]$ are considered. Priors bounds were selected to largely encompass reference values found in literature or according to thermodynamic stability criteria or physical limits. Given that $\mathcal{U}$ points out a uniform probability distribution, the Bayes theorem ultimately reads

$$
\mathcal{P}(\mathbf{q} \mid \mathbf{o}) \propto \Pi_{j} \mathcal{L}_{j} \mathcal{U}_{\mathbf{q}}, \quad j=N_{e},
$$

being $N_{e}$ the number of experiments carried out. The resulting Probability Density Functions (PDF) are reported in Fig. 6 (we recall that $\xi_{j}$ are, in order, $\left.P^{\mathrm{t}}, T^{\mathrm{t}}, P_{c r}, T_{c r}, \omega, \gamma\right)$. Generally, the mass of the posteriors is accumulated over the upper or the lower bounds. This reveals that inference process is trying to explore regions of the stochastic space which are outside the imposed prior bounds. Only the posterior of $\xi_{1}$, related to the total pressure $P^{\mathrm{t}}$ at the nozzle inlet, is fully contained within the prior range. In general, the Bayesian framework fails in finding a combination of values allowing the matching between numerics and experiments. Results suggest either an epistemic uncertainty underlying the computational model of the test section or biased experimental observations. 

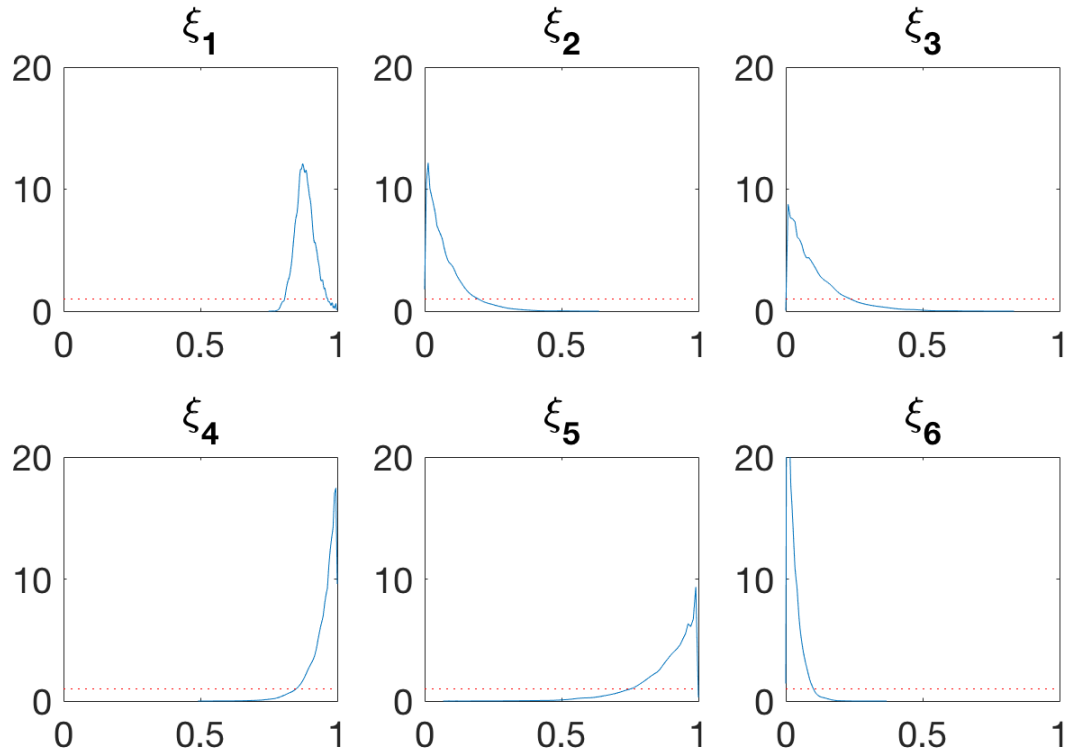

Fig. 6. posterior PDFs for the PR thermodynamic model parameters. The red line is the prior, the blue line the posterior.

Inference based on a synthetic data set i.e., observations are extrapolated from high-fidelity simulations, reveal that the considered experiment may not be suitable for the goal of inferring the Peng-Robinson model coefficients for MDM [20]. Indeed, it is virtually shown that, despite the variety of measurements that can possibly be obtained within the TROVA, little may be potentially learned. Nevertheless, the framework is returning substantial indications towards the development of future experiments. For instance, temperature measurements would be beneficial to the inference process, as their inclusion helps sharpening the posterior distributions of some unknowns. Moreover, in the considered thermodynamic conditions the problem is utterly dominated by the uncertainty over the value of total pressure at the domain inlet. Therefore, accurately controlling the flow inflow conditions is crucial. Though not easily achievable in practice, test conditions closer to the saturation curve, which foster more significant non-ideal effects, yield an improved output of the inference process.

\section{Conclusions}

This contribution reviews some of the advancements achieved in the frame of two EU-funded project (UTOPIAE and NSHOCK). Namely, we provide an overview about non-ideal oblique shock waves. The non-ideal effects described in Sec. 2 are relevant to many applications including, just to mention a few, engine intakes rockets, supersonic nozzle outflows, under-expanded jets and highly-loaded 
turbo-machinery stages. In particular, they may influence the pattern of the typical fish-tail shocks generated at the turbine stator blade training edge. Therefore, their understanding is of the utmost relevance for the future improvement of ORC turbine design.

In Sec 3 we review the first-ever experimental-based accuracy assessment of computational models for NICFD flows. Results reveal that all the considered non-ideal flows are fairly well simulated by the SU2 NICFD CFD solver. The variability of the numerical solution is limited to very small values, pointing out the robustness and the predictive character of the numerical tool. Nevertheless, numerical/experimental comparisons also reveal a mismatch increasing with the flow regime non-ideal behavior. The mismatch suggests that the CFD model of the TROVA test-rig could be further improved.

In Sec 4 we review a Bayesian framework for the inference of the materialdependent parameters entering complex equation of state for non-ideal flows. Results suggest that the available measurements are possibly biased or that the computational model includes some inherent uncertainties. Anyway, an analysis based on a synthetic data set reveals that the available observations (Mach and pressure measurements) would not bring substantial information to the inference process. The analysis shows that temperature measurements, though difficult to obtain, would instead be beneficial.

\section{Acknowledgements}

This research was partially funded by the UTOPIAE Marie Curie Innovative Training Network, H2020-MSCA-ITN-2016, Grant Agreement number 722734 and partially funded by the European Research Council under Grant ERC Consolidator 2013, project NSHOCK 617603. Numerical experiments presented in this paper were carried out using the PlaFRIM experimental testbed, supported by Inria, CNRS (LABRI and IMB), Université de Bordeaux, Bordeaux INP and Conseil Régional d'Aquitaine (see https://www.plafrim.fr/)

\section{References}

1. Bates, J.W., Montgomery, D.C.: Some numerical studies of exotic shock wave behavior. Phys. Fluids 11(2), 462$475 \quad$ (1999). $\quad$ https://doi.org/http://dx.doi.org/10.1063/1.869862, http://scitation.aip.org/content/aip/journal/pof2/11/2/10.1063/1.869862

2. Bethe, H.A.: The theory of shock waves for an arbitrary equation of state. Technical paper 545, Office Sci. Res. \& Dev. (1942)

3. Cinnella, P., Congedo, P., Parussini, L.: Quantification of thermodynamic uncertainties in real gas flows. International Journal of Engineering Systems Modelling and Simulation 2(1-2), 12-24 (2010)

4. Cinnella, P., Congedo, P., Pediroda, V., Parussini, L.: Sensitivity analysis of dense gas flow simulations to thermodynamic uncertainties. Physics of Fluids (23) (2011)

5. Colonna, P., der Stelt, T.P., Guardone, A.: FluidProp: A program for the estimation of thermophysical properties of fluids. Energy Technology Section, Delft University of Technology, The Netherlands (2005) 
6. Congedo, P., Corre, C., Martinez, J.M.: Shape optimization of an airfoil in a BZT flow with multiple-source uncertainties. Computer Methods in Applied Mechanics and Engineering 200(14), 216-232 (Aug 2011). https://doi.org/10.1016/j.cma.2010.08.006, http://linkinghub.elsevier.com/retrieve/pii/S0045782510002392

7. Congedo, P., Geraci, G., Abgrall, R., Pediroda, V., Parussini, L.: Tsi metamodelsbased multi-objective robust optimization. Engineering Computations (Swansea, Wales) 30(8), 1032-1053 (2013)

8. Cramer, M.S., Kluwick, A.: On the propagation of waves exhibiting both positive and negative nonlinearity. J. Fluid Mech. 142, 9-37 (1984)

9. Cramer, M.S., Sen, R.: Shock formation in fluids having embedded regions of negative nonlinearity. Phys. Fluids 29, 2181-2191 (1986)

10. Cramer, M.S., Sen, R.: Exact solutions for sonic shocks in van der Waals gases. Phys. Fluids 30, 377-385 (1987)

11. D'Angelo, S., Vimercati, D., Guardone, A.: A unified description of oblique waves in ideal and non-ideal steady supersonic flows around compressive and rarefactive corners. Acta Mechanics 229, 2585-2595 (2018). https://doi.org/10.1007/s00707018-2130-6

12. Economon, T.D., Mudigere, D., Bansal, G., Heinecke, A., Palacios, F., Park, J., Smelyanskiy, M., Alonso, J.J., Dubey, P.: Performance optimizations for scalable implicit RANS calculations with SU2. Computers \& Fluids 129, 146 - 158 (2016). https://doi.org/http://dx.doi.org/10.1016/j.compfluid.2016.02.003, http://www.sciencedirect.com/science/article/pii/S0045793016300214

13. Economon, T.D., Palacios, F., Copeland, S.R., Lukaczyk, T.W., Alonso, J.J.: SU2: An open-source suite for multiphysics simulation and design. AIAA Journal 54(3), 828-846 (2016/09/08 2015). https://doi.org/10.2514/1.J053813, http://dx.doi.org/10.2514/1.J053813

14. Geraci, G., Congedo, P., Abgrall, R., Iaccarino, G.: High-order statistics in global sensitivity analysis: Decomposition and model reduction. Computer Methods in Applied Mechanics and Engineering 301, 80 115 (2016). https://doi.org/http://dx.doi.org/10.1016/j.cma.2015.12.022, http://www.sciencedirect.com/science/article/pii/S0045782515004284

15. Gori, G.: Non-Ideal Compressible-Fluid Dynamics: Developing a Combined Perspective on Modeling, Numerics and Experiments. Ph.D. thesis, Politecnico di Milano (2019)

16. Gori, G., Guardone, A., Vitale, S., Head, A., Pini, M., Colonna, P.: Non-ideal compressible-fluid dynamics simulation with SU2: Numerical assessment of nozzle and blade flows for organic rankine cycle applications. In: 3rd International Seminar on ORC Power Systems. Brussels, Belgium (October 2015)

17. Gori, G., Vimercati, D., Guardone, A.: Non-ideal compressible-fluid effects in oblique shock waves. Journal of Physics: Conference Series 821(1), 012003 (2017), http://stacks.iop.org/1742-6596/821/i=1/a=012003

18. Gori, G., Vimercati, D., Guardone, A.: A numerical investigation of oblique shock waves in non-ideal compressible-fluid flows. In: 31st International Symposium on Shock Wavess. Nagoya, Japan (2017)

19. Gori, G., Zocca, M., Cammi, G., Spinelli, A., Congedo, P., Guardone, A.: Accuracy assessment of the non-ideal computational fluid dynamics model for siloxane mdm from the open-source su2 suite. European Journal of Mechanics - B/Fluids 79, 109 - 120 (2020). https://doi.org/https://doi.org/10.1016/j.euromechflu.2019.08.014, http://www.sciencedirect.com/science/article/pii/S099775461830712X 
20. Gori, G., Zocca, M., Guardone, A., Maître], O.L., Congedo, P.: Bayesian inference of thermodynamic models from vapor flow experiments. Computers \& Fluids 205, 104550 (2020). https://doi.org/https://doi.org/10.1016/j.compfluid.2020.104550, http://www.sciencedirect.com/science/article/pii/S0045793020301225

21. Gori, G., Zocca, M., Cammi, G., Spinelli, A., Guardone, A.: Experimental assessment of the open-source SU2 CFD suite for ORC applications. Energy Procedia 129(Supplement C), 256-263 (2017)

22. Guardone, A., Spinelli, A., Dossena, V.: Influence of molecular complexity on nozzle design for an organic vapor wind tunnel. ASME J. Eng. Gas Turb. Power 135, 042307 (2013)

23. Lambrakis, K.C., Thompson, P.A.: Existence of real fluids with a negative fundamental derivative $\Gamma$. Phys. Fluids 15(5), 933-935 (1972)

24. Le Maître, O., Knio, O.: Spectral Methods for Uncertainty Quantification. Scientific Computation, Springer Netherlands, 1 edn. (2010). https://doi.org/10.1007/978-90-481-3520-2

25. Menikoff, R., Plohr, B.J.: The Riemann problem for fluid flow of real material. Rev. Mod. Phys. 61(1), 75-130 (1989)

26. Merle, X., Cinnella, P.: Bayesian quantification of thermodynamic uncertainties in dense gas flows. Reliability Engineering \& System Safety 134(Supplement C), 305 - 323 (2015). https://doi.org/https://doi.org/10.1016/j.ress.2014.08.006, http://www.sciencedirect.com/science/article/pii/S0951832014001999

27. Nannan, N.R., Guardone, A., Colonna, P.: Critical point anomalies include expansion shock waves. Physics of Fluids 26(2), 021701 (2014). https://doi.org/http://dx.doi.org/10.1063/1.4863555, http://scitation.aip.org/content/aip/journal/pof2/26/2/10.1063/1.4863555

28. Nannan, N.R., Sirianni, C., Mathijssen, T., Guardone, A., Colonna, P.: The admissibility domain of rarefaction shock waves in the near-critical vapour-liquid equilibrium region of pure typical fluids. Journal of Fluid Mechanics 795, 241-261 (5 2016). https://doi.org/10.1017/jfm.2016.197

29. Palacios, F., Colonno, M.R., Aranake, A.C., Campos, A., Copeland, S.R., Economon, T.D., Lonkar, A.K., Lukaczyk, T.W., Taylor, T.W.R., Alonso, J.J.: Stanford University Unstructured $\left(\mathrm{SU}^{2}\right)$ : An open-source integrated computational environment for multi-physics simulation and design. AIAA Paper 2013-0287 51st AIAA Aerospace Sciences Meeting and Exhibit (January 2013)

30. Palacios, F., Economon, T.D., Aranake, A., Copeland, R.S., Lonkar, A., Lukaczyk, T., Manosalvas, D.E., Naik, R.K., Padron, S., Tracey, B., Variyar, A., Alonso, J.J.: Stanford university unstructured (SU2): Analysis and design technology for turbulent flows. AIAA Paper 2014-0243 52nd Aerospace Sciences Meeting (2014)

31. Peng, D.Y., Robinson, D.B.: A new two-constant equation of state. Ind. Eng. Chem. Fundam. 15, 59-64 (1976)

32. Pini, M., Spinelli, A., Dossena, V., Gaetani, P., Casella, F.: Dynamic simulation of a test rig for organic vapours (August 7-10 2011)

33. Pini, M., Vitale, S., Colonna, P., Gori, G., Guardone, A., Economon, T., Alonso, J., Palacios, F.: SU2: the open-source software for non-ideal compressible flows. vol. 821, p. $012013(2017)$

34. Roy, C.J., Oberkampf, W.L.: A comprehensive framework for verification, validation, and uncertainty quantification in scientific computing. Computer Methods in Applied Mechanics and Engineering 200(25), 2131 - 2144 (2011). https://doi.org/https://doi.org/10.1016/j.cma.2011.03.016, http://www.sciencedirect.com/science/article/pii/S0045782511001290 
35. Sanchez, R., Kline, H., Thomas, D., Variyar, A., Righi, M., Economon, D.T., Alonso, J.J., Palacios, R., Dimitriadis, G., Terrapon, V.: Assessment of the fluidstructure interaction capabilities for aeronautical applications of the open-source solver SU2. ECCOMAS, VII European Congress on Computational Methods in Applied Sciences and Engineering, Crete Island, Greece (2016)

36. Spinelli, A., Guardone, A., Cozzi, F., Carmine, M., Cheli, R., Zocca, M., Gaetani, P., Dossena, V.: Experimental observation of non-ideal nozzle flow of siloxane vapor mdm. In: 3rd International Seminar on ORC Power Systems, Brussels, Belgium, 12-14 October (2015)

37. Spinelli, A., Pini, M., Dossena, V., Gaetani, P., Casella, F.: Design, simulation, and construction of a test rig for organic vapours. ASME J. Eng. Gas Turb. Power 135, 042303 (2013)

38. Spinelli, A., Cammi, G., Zocca, M., Gallarini, S., Cozzi, F., Gaetani, P., Dossena, V., Guardone, A.: Experimental observation of non-ideal expanding flows of siloxane mdm vapor for orc applications. Energy Procedia 129, 1125 - 1132 (2017)

39. Spinelli, A., Cozzi, F., Zocca, M., Gaetani, P., Dossena, V., Guardone, A.: Experimental investigation of a non-ideal expansion flow of siloxane vapor MDM. In: Proceedings of the ASME 2016 Turbo Expo, Soul. No. GT2016-57357 (2016)

40. Thol, M., Dubberke, F.H., Baumhögger, E., Vrabec, J., Span, R.: Speed of sound measurements and fundamental equations of state for octamethyltrisiloxane and decamethyltetrasiloxane. Journal of Chemical \& Engineering Data 62(9), 26332648 (2017)

41. Thompson, P.A.: A fundamental derivative in gasdynamics. Phys. Fluids 14(9), 1843-1849 (1971)

42. Thompson, P.A.: Compressilbe Fluid Dynamics. McGraw-Hill (1988)

43. Thompson, P.A., Lambrakis, K.C.: Negative shock waves. J. Fluid Mech. 60, 187208 (1973)

44. Vimercati, D., Gori, G., Spinelli, A., Guardone, A.: Non-ideal effects on the typical trailing edge shock pattern of ORC turbine blades. Energy Procedia 129(Supplement C), 1109-1116 (2017)

45. Vimercati, D.: Non-Ideal Steady Supersonic Flows. Ph.D. thesis, Politecnico di Milano (2019)

46. Vimercati, D., Gori, G., Guardone, A.: Non-ideal oblique shock waves. Journal of Fluid Mechanics 847, 266-285 (2018). https://doi.org/10.1017/jfm.2018.328

47. Vitale, S., Gori, G., Pini, M., Guardone, A., Economon, T.D., Palacios, F., Alonso, J.J., Colonna, P.: Extension of the SU2 open source CFD code to the simulation of turbulent flows of fluids modelled with complex thermophysical laws. In: $22^{\text {nd }}$ AIAA Computational Fluid Dynamics Conference. No. AIAA Paper 2760 (2015)

48. Zeld́ovich, Y.B.: On the possibility of rarefaction shock waves. Zh. Eksp. Teor. Fiz. 4, 363-364 (1946)

49. Zocca, M.: Experimental observation of supersonic non-ideal compressible-fluid flows. Ph.D. thesis, Politecnico di Milano (2018)

50. Zocca, M., Guardone, A., Cammi, G., Cozzi, F., Spinelli, A.: Experimental observation of oblique shock waves in steady non-ideal flows. Exp Fluids 60, 101 (2019). https://doi.org/https://doi.org/10.1007/s00348-019-2746-x 